\title{
LXIX. On quaternions; or on a new system of imaginaries in algebra
}

\section{Professor Sir William Rowan Hamilton LL.D. V.P.R.I.A. F.R.A.S.}

To cite this article: Professor Sir William Rowan Hamilton LL.D. V.P.R.I.A. F.R.A.S. (1847) LXIX. On quaternions; or on a new system of imaginaries in algebra, Philosophical Magazine Series 3, 30:203, 458-461, DOI: 10.1080/14786444708645426

To link to this article: http://dx.doi.org/10.1080/14786444708645426

册 Published online: 30 Apr 2009.

Submit your article to this journal $\lceil\pi$

Џll Article views: 5

Q View related articles $₫$

4 Citing articles: 1 View citing articles 진 


\section{[ 4558 ]}

LXIX. On Quaternions; or on a Nerw System of Imaginaries in Algebra. By Professor Sir Wiluin liowan Hamilton, LL.D., V.P.R.I.A., F.R.A.S., Corresponding Member of the Institute of France, and of other Scientific Societies in British and Foreign Countries, Andrewes' Professor of Astronomy in the University of Dublin, and Royal Astronomer of Ireland.

[Continued from vol, xxix. p. 328.]

29. $F$ we denote by $\alpha$ and $\beta$ two constant vectors, and by $\rho$ a variable vector, all drawn from one common origin; if also we denote by $u$ and $v$ two variable scalars, depending on the foregoing vectors $\alpha, \beta, \rho$ by the relations

$$
\begin{aligned}
& u=2 \mathrm{~S} \cdot \alpha \rho=\alpha \rho+\rho \alpha ; \\
& \left.v^{2}=-4(\mathrm{~V} \cdot \beta \rho)^{2}=-(\beta \rho-p \beta)^{2} ;\right\} . . .
\end{aligned}
$$

we may then represent the central surfaces of the second degree by equations of great simplicity, as follows :-

An ellipsoid, with three unequal axes, may be represented by the equation

$$
u^{2}+v^{2}=1 \text {. . . . . . }
$$

One of its circumscribing cylinders of revolution has for equation

$$
v^{2}=1 ; . . . . . .
$$

the plane of the ellipse of contact is represented by

$$
u=0 ; . . . \text {. . . . }
$$

and the system of the two tangent planes of the ellipsoid, parallel to the plane of this ellipse, by

$$
u^{2}=1 \text {. . . . . . . . }
$$

A hyperboloid of one sheet, touching the same cylinder in the same sheet, is denoted by the equation

$$
u^{2}-v^{2}=-1 ; \text {. . . . . }
$$

its asymptotic cone by

$$
u^{2}-v^{2}=0 \text {; }
$$

and a hyperboloid of two sheets, with the same asymptotic cone (7.), and with the two tangent planes (5.), is represented by this other equation,

$$
u^{2}-v^{2}=1 . \text {. . . . . . }
$$

By changing $\rho$ to $\rho-\gamma$, where $\gamma$ is a third arbitrary but constant vector, we introduce an arbitrary origin of vectors, or an arbitrary position of the centre of the surface, as referred to such an origin. And the general problem of determining that individual surface of the second degree (supposed to have a centre, until the calculation shall show in any particular question that it has none), which shall pass through nine given 
points, may thus be regarded as equivalent to the problem of finding three constant vectors, $\alpha, \beta, \gamma$, which shall, for nine given values of the variable vector $\rho$, satisfy one equation of the form

$$
\{\alpha(p-\gamma)+(p-\gamma) \alpha\}^{2} \pm\{\beta(p-\gamma)-(p-\gamma) \beta\}^{2}= \pm 1 ; .
$$

with suitable selections of the two ambiguous signs, depending on, and in their turn determining, the particular species of he surface

30. The equation of the ellipsoid with three unequal axes, referred to its centre as the origin of vectors, may thus be presented under the following form (which was exhibited to the Royal Irish Academy in December 184.5):

$$
\left(\alpha \rho+\rho \alpha^{2}\right)-(\beta p-p \beta)^{2}=1 \text {; . . . . }
$$

and which decomposes itself into two factors, as follows :

$$
(\alpha p+p \alpha+\beta p-\rho \beta)(\alpha p+p \alpha-\beta p+p \beta)=1 . .
$$

These two factors are not only separately linear with respect to the variable vector $\rho$, but are also (by art. 20, Phil. Mag. for July 1846) conjugate quaternions; they have therefore a common tensor, which must be equal to unity, so that we may write the equation of the ellipsoid under this other form,

$$
\mathrm{T}(\alpha \rho+\rho \alpha+\beta \rho-\rho \beta)=1 ; . . . \quad . \quad \text {. }
$$

if we use, as in the 19th article, Phil. Mag., July 1846, the characteristic $\mathrm{T}$ to denote the operation of taking the tensor of a quaternion. Let $\sigma$ be an auxiliary vector, connected with the vector $\rho$ of the ellipsoid by the equation

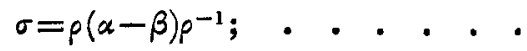

we shall then have, by (3.), and by the general law for the tensor of a product,

but also

$$
\mathbf{T}(\alpha+\beta+\sigma) \cdot \mathbf{T}_{\rho}=1 ; . . . .
$$

$$
(\alpha-\beta+\sigma) p=(\alpha-\beta) p+p(\alpha-\beta)
$$

where the second member is scalar; therefore, using the characteristic $U$ to denote the operation of taking the versor of a quaternion, as in the same art. 19, we have the equation

$$
\mathrm{U}(\alpha-\beta+\sigma) \cdot \mathrm{U}_{\rho}=\mp 1 ; . . . \cdot
$$

and the dependence of the variable vector $\rho$ of the ellipsoid on the auxiliary vector $\sigma$ is expressed by the formula

$$
\rho= \pm \frac{\mathrm{U}(\alpha-\beta+\sigma)}{\mathrm{T}(\alpha+\beta+\sigma)^{\circ}} \cdot \text {. . . . }
$$

Besides, the length of this auxiliary vector $\sigma$ is constant, and equal to that of $\alpha-\beta$, because the equation (4.) gives

$$
\mathrm{T} \sigma=\mathrm{T}(\alpha-\beta) ; . \cdot \cdot \cdot \cdot
$$


we may therefore regard $\alpha-\beta$ as the vector of the centre $C$ of a certain auxiliary sphere, of which the surface passes through the centre A of the ellipsoid; and may regard the vector $\alpha-\beta+\sigma$ as a variable and auxiliary guide-chord $\mathrm{AD}$ of the same guide-sphere, which chord determines the (exactly similar or exactly opposite) direction of the variable radius vector $\mathrm{AE}$ (or $\rho$ ) of the ellipsoid. At the same time, the constant vector $-2 \beta$, drawn from the same constant origin as before, namely the centre $A$ of the ellipsoid, will determine the position of a certain fixed point $B$, having this remarkable property, that its distance from the extremity $D$ of the variable guide-chord drawn from $\mathrm{A}$, will represent the reciprocal of the length of the radius vector $\rho$, or the proximity $(\mathrm{AE})^{-1}$ of the point $E$ on the surface of the ellipsoid to the centre (the use of this word "proximity" being borrowed from Sir John Herschel). Supposing then, for simplicity, that the fixed point $B$ is external to the fixed sphere, which does not essentially diminish the generality of the question; and taking, for the unit of length, the length of a tangent to that sphere from that point; we may regard $\mathrm{AE}$ and $\mathrm{BD}^{\prime}$ as two equally long lines, or may write the equation

$$
\overline{\mathrm{AE}}=\overline{\mathrm{BD}^{\prime}}, . . . \cdot \text {. }
$$

if $\mathrm{D}^{\prime}$ be the other point of intersection of the straight line $\mathrm{BD}$ with the sphere.

31. Hence follows this very simple construction* for an ellipsoid (with three unequal axes), by means of a sphere and an external point, to which the author was led by the foregoing process, but which may also be deduced from principles more generally known. From a fixed point $A$ on the surface of a sphere, draw a variable chord $A D$; let $\mathrm{D}^{\prime}$ be the second point of intersection of the spheric surface with the secant $B D$, drawn to the variable extremity $D$ of this chord $A D$ from a fixed external point $B$; take the radius vector $A E$ equal in length to $\mathrm{BD}^{\prime}$, and in direction either coincident with, or opposite to, the chord $\mathrm{AD}$; the locus of the point $\mathrm{E}$, thus constructed, roill be an ellipsoid, which will pass through the point B.

* This construction has already been printed in the Proceedings of the Royal Irish Academy for 1846 ; but it is conceived that its being reprinted here may be acceptable to some of the readers of the London, Edinburgh, and Dublin Philosophical Magazine; in which periodical (namely in the Number for July 1844) the first printed publication of the fundamental equations of the theory of quaternions $\left(i^{2}=j^{2}=k^{2}=-1, i j=k, j k=i, k i=j\right.$, $j i=-k, k j=-i, i k=-j$ ) took place, although those equations had been communicated to the Royal Irish Academy in November 1843, and had been exhibited at a meeting of the Council during the preceding month., 
32. We may also say that if of a quadrilateral $\left(\mathrm{ABED}^{\prime}\right)$, of which one side $(\mathrm{AB})$ is given in length and in position, the two diagonals $\left(\mathrm{AE}, \mathrm{BD}^{\prime}\right)$ be equal to each other in length, and intersect (in D) on the surface of a given sphere (with centre C), of which a chord $\left(\mathrm{AD}^{\prime}\right)$ is a side of the quadrilateral adjacent to the given side (AB), then the other side (BE), adjacent to the same given side, is a chord of a given ellipsoid. The form, position, and magnitude of an ellipsoid (with three unequal axes), may thus be made to depend on the form, position, and magnitude of a generating triangle ABC. 'Two sides of this triangle, namely $\mathrm{BC}$ and $\mathrm{CA}$, are perpendicular to the troo planes of circular section; and the third side $\mathrm{AB}$ is perpendicular to one of the two planes of circular projection of the ellipsoid, because it is the axis of revolution of one of the two circumscribed circular cylinders. This triple reference to circles is perhaps the cause of the extreme facility with which it will be found that many fundamental properties of the ellipsoid may be deduced from this mode of generation. As an example of such deduction, it may be mentioned that the known proportionality of the difference of the squares of the reciprocals of the semiaxes of a diametral section to the product of the sines of the inclinations of its plane to the two planes of circular section, presents itself under the form of a proportionality of the same difference of squares to the rectangle under the projections of the two sides $\mathrm{BC}$ and $\mathrm{CA}$ of the generating triangle on the plane of the elliptic section.

[To be continued.]

LXX. Observations on the Analysis of the Spectrum by Absorption. By Sir David Brewster, K.H., D.C.L., F.R.S., and V.P.R.S. Ed.*

I $\mathrm{N}$ a paper On the Production of Light and Heat by $1 \mathrm{Dr}$. Draper, published in the last Number of this Journal, the ingenious author has made some observations on my Analysis of the Solar Spectrum, which it is necessary that I should notice. While he admits that I have "shown that red, yellore, blue, and consequently white light exist in every part of the spectrum," he ascribes this result to the non-coincidence, or the overlapping of the colours of the several spectra formed on a screen by the rays falling near the edge, and those falling near the back of a prism with a refracting face of considerable magnitude. "In such a spectrum," he says, "there must undoubtedly be a general commixture of the rays; but may we not fairly inquire whether, if an elementary prism were

* Communicated by the Author. 